\title{
'Near miss' sudden infant death and obstructive apnoea
}

\author{
K P DUNNE, M MCKAY, AND T G MATTHEWS \\ Department of Paediatrics, Rotunda Hospital, Dublin, Eire
}

\begin{abstract}
SUMMARY A 4 month old girl presented with a 'near miss' sudden infant death episode for which no cause was found. Obstructive sleep apnoea syndrome subsquently developed. All symptoms ceased after adenoidectomy at age 9 months.
\end{abstract}

'Near miss' sudden infant death syndrome (SIDS) is a diagnosis based on a history of a child found apparently lifeless and requiring vigorous resuscitation to revive him. Investigations fail to reveal a convincing cause. Obstructive sleep apnoea syndrome in children and adults is rarely diagnosed in the United Kingdom compared with the experience of the United States. ${ }^{1}$ We report a case of 'near miss' SIDS who subsequently developed obstructive sleep apnoea syndrome.

\section{Case report}

A 4 month old girl was admitted for evaluation after an unexplained apnoeic episode (lasting 15-20 seconds) at age 11 weeks. While awake, she suddenly became apnoeic and stiff, and the mother thought she was about to die. There was no history of difficulty in breathing or struggling. She was resuscitated by vigorous back slapping and was normal in a few minutes. There had been no previous episodes and her breathing was otherwise normal. She was the third child, a full term normal delivery with birth weight $3300 \mathrm{~g}$ and length $50 \mathrm{~cm}$, after an uneventful pregnancy. There were no neonatal problems. Both the child's mother and grandfather had had tonsillectomy and adenoidectomy as children and the grandfather still snores. There was no family history of SIDS.

Clinical examination yielded normal results, except that weight $(5.28 \mathrm{~kg})$ and length $(58.1 \mathrm{~cm})$ were both less than 10th centile. All investigations (full blood count, electrolyte and calcium concentrations, electrocardiogram, and electroencephalogram) yielded completely normal results. Overnight cardiorespiratory monitoring (512 Neonatal monitor, Corometrics Medical Systems Inc) showed occasional periodic breathing but no prolonged episodes of apnoea ( $>15$ seconds) or bradycardia ( $<90$ beats/minute). End tidal carbon dioxide in quiet sleep was $5.0 \mathrm{kPa}$ and her end tidal carbon dioxide on breathing $12 \%$ carbon dioxide could be raised to $7.5 \mathrm{kPa}$ before arousal from sleep. She was diagnosed as a case of 'near miss' SIDS and received no treatment but was kept under regular outpatient review.

There were no further attacks, but from age 6 months her mother became increasingly concerned with the child's breathing when asleep. She had intermittent snoring and, despite no medical background, the mother gave a clear history of episodes of complete upper airway obstruction. The child would go quiet, the chest would move up and down, but no air was going in 'as if some one was pinching her nose'. Typically, the infant then started to cry, took a few deep gasping breaths, and woke up. This occurred two to three times a night initially, becoming almost continuous when she was seen in the outpatients ward at 8 months, causing total disruption of sleep for mother and child. There was no history of sweating with these episodes.

Examination revealed a normal child (weight $7 \cdot 25$ $\mathrm{kg}(<10$ th centile), height $64.0 \mathrm{~cm}(<3$ rd centile) $)$ with no signs of right ventricular hypertrophy and no evidence of upper airways obstruction when awake. Investigations included a normal electrocardiogram and chest $x$ ray film, but a lateral neck $x$ ray film showed adenoidal hypertrophy. Cardiorespiratory monitoring was not repeated. After discussion with the parents laryngoscopy and bronchoscopy were performed under anaesthesia, proceeding to adenoidectomy when this confirmed pronounced adenoidal hypertrophy. Since the operation she has had no 'near miss' episodes and her breathing is normal. At age 1 year her height is now $73.3 \mathrm{~cm}$ and weight $8.80 \mathrm{~kg}$ (both 10 th to 25 th centile) and physical examination and development is completely normal.

\section{Discussion}

Obstructive sleep apnoea syndrome remains an uncommon diagnosis in medical practice in the UK. The pathophysiology of the syndrome is not certain, but floppy laryngeal walls combined with narrowing of the nasopharyngeal airway-for example, by adenoidal enlargement-may predispose to upper airway collapse. ${ }^{1}$ Chronic obstruction may cause 
apnoea, hypercapnoea, and hypoxia. Cor pulmonale, neurological deficits, learning difficulty, failure to thrive, ${ }^{2}$ and short stature $^{3}$ may result. Adenoidectomy results in a 'catch up' of weight and height $t^{23}$ as in our patient. The fact that questions about sleep or sleep problems are not as yet part of the routine medical questioning may partly explain this clinical 'blind spot'. Even though all cases are symptomatic, however, there is often considerable delay in referral. ${ }^{2}$ Clinical examination when awake may yield completely normal results, and it is important to assess the child when asleep.

The association between 'near miss' SIDS and the subsequent development of obstructive sleep apnoea syndrome is a rare one, and to our knowledge there are only 12 reported cases (including one child who died of SIDS), all published in the US. ${ }^{4-6}$ The connection is unclear, but Guilleminault postulates that a small posterior airway at the level of the tongue may predispose to apnoea and this may be familial. ${ }^{6}$ In our case there was no history or clinical evidence of airway obstruction in the initial 'near miss' episode. The initial polygraphic recording was normal but does not reliably exclude airway obstruction in the absence of data relating to nasal air flow. The association is, however, important. Obstructive sleep apnoea syndrome must be excluded as a treatable cause of 'near miss' SIDS. A thorough history, examination of the child when asleep, and a lateral neck $x$ ray film are essential as well as cardiorespiratory monitoring. It must be noted, however, that a negative lateral neck $x$ ray does not exclude adenoidal hypertrophy. ${ }^{2}$ An otorhinolaryngological examination is mandatory if obstructive sleep apnoea is suspected on history.

Obstructive sleep apnoea is one possible cause for the failure to gain weight sometimes noted before deaths through SIDS. Guilleminault noted four siblings had died from SIDS in the five families described with a small posterior airway. ${ }^{6}$ Such an association is speculative and obviously does not explain most cases of SIDS. Physicians must be aware, however, that children with 'near miss' SIDS may subsequently develop obstructive sleep apnoea and that this can occur at an early age. Further follow up studies may show that this association is more common than had been previously thought.

We thank Mr Collins (Consultant Ears, Nose, and Throat Surgeon) and Miss Audrey Dixon. Dr K Dunne is funded jointly by the Friends of the Rotunda and the Irish Sudden Infant Death Association.

\section{References}

1 Anonymous. Snoring and sleepiness [Editorial]. Lancet 1985;ii:925-6.

2 Brouillette RT, Fernbach SK, Hunt CE. Obstructive sleep apnoea in infants and children. $J$ Pediatr 1982;100:31-40.

${ }^{3}$ Bate TWP, Price DA, Holme CA, McGucken RB. Short stature caused by obstructive sleep apnoea during sleep. Arch Dis Child 1984;59:78-80.

${ }^{4}$ Guilleminault C, Ariagno RL, Forno LS, Nagel L, Baldwin R, Owen M. Obstructive sleep apnoea and "near-miss" for S.I.D.S.: 1. Report of an infant with sudden death. Pediatrics 1979;63:837-43.

${ }^{5}$ Guilleminault C, Souquet M, Ariagno RL, Korobkin R, Simmons FB. Five cases of near-miss sudden infant death syndrome and development of obstructive sleep apnoea syndrome. Pediatrics 1984;73:71-8.

${ }^{6}$ Guilleminault C, Powell N, Heldt G, Riley R. Small upper airway in near-miss sudden infant death syndrome infants and their families. Lancet 1986;i:402-7.

Correspondence to Dr T G Matthews, Department of Paediatrics, Rotunda Hospital, Dublin 1, Eire.

Received 20 June 1986 